\title{
Aplicaciones Móviles para el Estudio de la Anatomía Humana
}

\author{
Mobile Applications for the Study of Human Anatomy
}

Jaime Esteban Lucero Mueses ${ }^{1} \&$ Oscar Andrés Álzate Mejía ${ }^{2}$

\begin{abstract}
LUCERO, M. J. E. \& ÁLZATE, M. O. A. Móviles para el estudio de la anatomía humana. Int. J. Morphol., 38(5):1365-1370, 2020.
RESUMEN: El aprendizaje a través de aplicaciones móviles (Apps) es un tema de gran importancia que está asociado a interactuar con la realidad virtual. Pese a la gran cantidad de Apps disponibles, no existe información suficiente que oriente acerca de la utilidad en la didáctica de la anatomía. Es por ello que este trabajo pretende evaluar el uso de aplicaciones móviles en el estudio de la Anatomía humana. Se realizó la búsqueda de aplicaciones para el estudio de la anatomía humana, utilizando las plataformas de descarga App Store y Google Play. Se jerarquizaron las Apps dando importancia a las que contaran con un mayor número de personas que la hubieran valorado y a la puntuación que le otorgaron sus usuarios. Se incorporaron Apps al estudio de la Anatomía humana en estudiantes de salud de universidades de la ciudad de Manizales (Colombia). Se aplicó un instrumento para evaluar la utilidad en el aprendizaje de las diferentes estructuras humanas. La búsqueda dio como resultado 113 aplicaciones, de las cuales 70 son gratuitas y 43 que tienen un costo para su descarga. De éstas, Anatomy quiz es la que más prefieren los estudiantes al momento de estudiar anatomía. La utilización de Apps para el estudio de la anatomía humana prepara a estudiantes para enfrentarse a una estructura real; se cuenta con especímenes ilimitados para una disección virtual y desarrolla habilidades espaciales. Conocer las Apps disponibles para estudio del cuerpo humano aporta a una mejor didáctica de la Anatomía.
\end{abstract}

PALABRAS CLAVE: Aplicaciones móviles; Anatomía; Aprendizaje; Realidad virtual (MeSH).

\section{INTRODUCCIÓN}

Las tecnologías de información y comunicación (TICs) son una herramienta que facilita el aprendizaje de temas de alta complejidad, la sociedad necesita personas preparadas con competencias en su manejo dentro de los distintos ámbitos profesionales (López García \& Morcillo Ortega, 2007). Sin embargo, algunos docentes no son conscientes de la gran utilidad que se puede dar a estas importantes herramientas (Raikos \& Waidyasekara, 2014).

El aprendizaje a través de aplicaciones móviles (Apps) es un tema de gran importancia, las personas puede tener acceso desde un equipo móvil que puede llevar a cualquier parte (Basogain et al., 2015). Una App permite interacción, un mejor aprendizaje en un ámbito completamente virtual y de fácil acceso. La mayoría de las aplicaciones complementan los métodos tradicionales de enseñanza (de Pedro Carracedo \& Martínez Méndez, 2012).

De manera específica, gran cantidad de estudiantes tienen dificultad para entender temas asociados con el funcionamiento del cuerpo. Las clases de Anatomía se orientan más hacia la teoría, no se da la importancia requerida a las prácticas (Alzate-Mejía \& Tamayo-Alzate, 2019). Los estudiantes reclaman sobre la necesidad de ellas para complementar la teoría vista en clase. Por su parte, es evidente el auge que ha tenido la utilización de nuevas tecnologías para la enseñanza de la anatomía (Farfán et al., 2016). La disección se está trasladando a un escenario virtual lo que ofrece así otras oportunidades para recrear el cuerpo. Es así que se está proponiendo complementar las prácticas de la disección por sesiones frente a computadoras o teléfonos móviles (Johnson et al., 2012).

Es necesario entonces conocer las Apps que pueden ser útiles para una mejor comprensión de las temáticas vistas en clase de Anatomía. Por tal razón, en este trabajo se hizo una búsqueda exhaustiva de Apps que están encaminadas al estudio del cuerpo humano, para descubrir, clasificar y obtener las que realmente le serán de gran ayuda a un estudiante que encamina su profesión en áreas de la salud. El objetivo entonces fue evaluar el uso de aplicaciones móviles en el estudio de la Anatomía humana.

\footnotetext{
${ }^{1}$ Programa de Ingeniería Biomédica, Universidad Autónoma de Manizales, manizales, Colombia.

${ }^{2}$ Departamento de Ciencias Básicas Biológicas, Universidad Autónoma de Manizales, Manizales, Colombia.
} 


\section{MATERIAL Y MÉTODO}

Se hizo una investigación cualitativa descriptiva que se basó en tres momentos específicos:

I. Búsqueda y clasificación de Apps en Anatomía. Se realizó la búsqueda de aplicaciones para el estudio de la anatomía humana, utilizando las plataformas de descarga App Store para dispositivos con sistema operativo IOs y Google Play para dispositivos con sistema operativo Android, para ello se utilizaron términos como: "anatomía", "huesos", "Anatomy", "aprendizaje anatomía", "cuerpo humano" y "músculos".

Encuesta sobre la urifidad de Appos para el estudio de la anatomis

USO DE APLICACIONES MOVILES (APPS) PARA EL ESTUDIO DE LA ANATOMIA HUMANA

Estimados estudiantes, la presente encuesta pretende evidenciar la utilidad que tiene el uso de Apps en el estudio de la Anatomia Humana. es por ello que le solicitamos responder las siguientes preguntas:

Nombres (opcional):

Universidad y programa:

1. ¿Considera útil el uso de Apps para el estudio de la Anatomia humana? $\underline{\text { SI - NO }}$

2. ¿Cuáles considera que son las ventajas de estudiar anatomía con Apps? a.

b.

c.

3. ¿Cuáles obstáculos o desventajas ha identificado cuando estudia Anatomia con Apps? a.

b.

c.

4. ¿Cuál de las Apps que utiliza para estudiar anatomia le parece la mejor? ¿por qué?

5. ¿Le recomendaria a un compan̂ero estudiar Anatomía con Apps? ¿Cuál? ¿por qué?

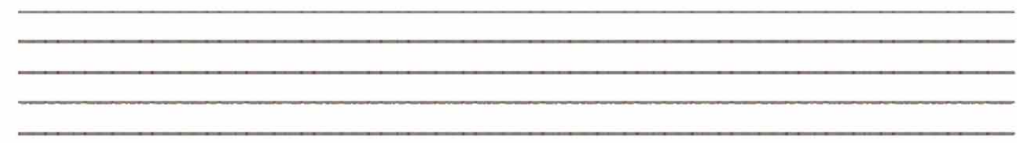

'Gracias por su valioso aporte!

Semillero de investigacioin Morfi - Universidad Aundnoma be Mamizales

Fig. 1. Formulario de encuesta para analizar el uso de aplicaciones móviles en el estudio de la anatomía humana.
Después de obtener una gran cantidad de aplicaciones se procedió observar detalladamente las descripciones de cule modo de descarga es gratuito y las Apps que reApps encontradas dado importancia las que contaran con un mayor número de personas que la han valorado y a la puntuación que le han otorgado sus respectivos usuarios.

II. Incorporación y selección de Apps. 53 estudiantes de terapia de la Universidad Autónoma de Manizales y diantes de Medicina de la Universidad de Manizales en Colombia, durante el segundo semestre del año 2019, incorporaron Apps al estudio de la Anatomía en las diferentes regiones del cuerpo humano.

III. Validación de Apps en el estudio de la Anatomía. Se diseñó y aplico un instrumento (Fig. 1) a los estudiantes que mostraron la percepción y utilidad de las Apps al estudio de la Anatomía humana. Se totalizaron las opiniones y de este totalse obtuvieron porcentajes

\section{RESULTADOS}

Apps encontradas para el estudio de la Anatomía humana. En la búsqueda de aplicaciones móviles para el estudio de la anatomía humana realizada hasta octubre de 2019 se encontraron 113 aplicaciones sin contar las orientadas al aprendizaje de niños, en este trabajo se incluyeron sólo aplicaciones enfocadas a estudiantes de básica secundaria y universitarios, de las cuales 70 son de manera gratuita y 43 apps que tienen un costo para poder llevar acabo su descarga. Algunas de estas apps sólo son disponibles en iOS, otras sólo en Android y otras son disponibles en los dos sistemas operativos. Con la información obtenida se elaboraron dos tablas, una para las aplicaciones sin costo, donde se muestra su link de descarga, el número de valoraciones que los usuarios le han dado y su respectiva puntuación la cual abarca de 0 a 5 (Tabla I). La otra muestra las aplicaciones con costo donde se muestran su respectivo link de descarga, el número de usuarios que la 
Tabla I. Aplicaciones móviles gratuitas para el estudio de la Anatomía humana:

\begin{tabular}{|c|c|c|c|}
\hline Nombre & Link De Desc arga & Valoraciones & Puntuacion \\
\hline 1. A natomy Learning - 3D Atlas & https://n9.cl/nn5d & 81064 & 4,6 \\
\hline 2. Ó rganos 3D (anatomía) & https://n9.cl/rcg4 & 34534 & $\underline{4,5}$ \\
\hline 3. $\mathrm{V}$ isual Anatomy Free & https://n9.cl/z4h0 & $\underline{29577}$ & $\underline{4,4}$ \\
\hline 4. $\mathrm{S}$ istema Ós eo 3D(anatomía) & https://n9.cl/w6ap2 & 17220 & 4,5 \\
\hline 5. $\mathrm{T}$ each Me Anatomy & https://n9.cl/4h54 & $\underline{14546}$ & $\underline{4,6}$ \\
\hline 6. 3 D Bones and Orga ns (anatomy) & https://n9.cl/rrps & 12746 & $\underline{4,4}$ \\
\hline 7. G ray's Anatomy Atlas & https://n9.cl/tqm4 & 12233 & 4,6 \\
\hline 8. 3 D Brain & https://n9.cl/2iji & 6223 & 4.2 \\
\hline 9. M úsculos | Esqueleto 3D Atlas De Anatomía: & https://n9.cl/925x & $\underline{5680}$ & $\underline{3.7}$ \\
\hline 10. B ioDigital Human - 3D Anatomy & $\mathrm{https}: / / \mathrm{n} 9 . \mathrm{cl} / \mathrm{g} 83 \mathrm{~m}$ & $\overline{4372}$ & $\overline{4,6}$ \\
\hline 11. D aily Anatomy: Flashcard Quizzes to Learn Anatomy & https://n9.cl/1d4r & 3389 & 4.5 \\
\hline 12. $\mathrm{v}$ isual Anatomy Lite & https://n9.cl/u5lg & $\underline{2100}$ & $\underline{4,6}$ \\
\hline 13. A natomyka - 3D Human Anatomy Atlas & https://n9.cl/u1bo & 1927 & 4.6 \\
\hline 14. E -Anatomy & https://n9.cl/2zg2 & 1856 & 4,6 \\
\hline 15. M y Organs Anatomy & https://n9.cl/1xf0 & 1707 & $\underline{4,1}$ \\
\hline 16. C omplete Anatomy Platform 2020 & https://n9.cl/mt30 & $\overline{1515}$ & 4.6 \\
\hline 17. A natomy Quiz & https://n9.cl/syad & 1094 & 4,5 \\
\hline 18. A natomy \& Physiology & https://n9.cl/ruqp & 732 & $\underline{4.4}$ \\
\hline 19. D igestive System Anatomy & https://n9.cl/m14g & 681 & 4,2 \\
\hline 20. F isiología Humana & https://n9.cl/stey & 521 & 3,7 \\
\hline : & $\vdots$ & $\vdots$ & $\vdots$ \\
\hline \multirow[t]{2}{*}{$:$} & $:$ & $:$ & $:$ \\
\hline & : & & \\
\hline 24. A natomist - Anatomía Cuestionario Juego & https://n9.cl/q15r & $\underline{218}$ & $\underline{4,5}$ \\
\hline
\end{tabular}

ha valorado, la puntuación que también abarca de 0 a 5 y el costo de la app que se muestra en dólares y euros respectivamente (Tabla II). Se ordenaron las apps dependiendo de las valoraciones que hasta la fecha han dado los usuarios.
De las 70 apps gratuitas, 47 tuvieron valoración. De las 43 apps con costo, 29 tuvieron valoración. Se seleccionaron las mejores 20 de cada categoría y son las que se muestran en las respectivas tablas.

Tabla II. Aplicaciones móviles con costo para el estudio de la Anatomía humana.

\begin{tabular}{|c|c|c|c|c|}
\hline Nombre & Link De Descarga & Valoraciones & Puntuacion & Costo \\
\hline 1. E ssential Anatomy 5 & https://n9.cl/gltz & 8200 & 4,8 & $\$ 14.99$ \\
\hline 2. $\mathrm{H}$ uman Anatomy Atlas 2020 & $\overline{\mathrm{https}: / / \mathrm{n} 9 . \mathrm{cl} / \mathrm{c} 8 \mathrm{aq}}$ & 7500 & 4,9 & $\$ 24.99$ \\
\hline 3. i Muscle 2 & https://n9.cl/0lu3 & 2650 & 3,7 & $\$ 6.00$ \\
\hline 4. $\mathrm{V}$ isual Muscles 3D & https://n9.cl/i4 1w & 470 & 4,4 & $\$ 7.00$ \\
\hline 5. M uscle Trigger Points & $\overline{\mathrm{https}: / / \mathrm{n} 9 . \mathrm{cl} / \mathrm{upfv}}$ & 257 & 4,7 & $\$ 2.99$ \\
\hline 6. 3 D Anatomy & https://n9.cl/q51p & 175 & 4,6 & $\$ 3.99$ \\
\hline 7. Learn Muscles: Anatomy & https://n9.cl/wzlv & 129 & 4,4 & $\$ 2.99$ \\
\hline 8. A natomyMapp & $\overline{\mathrm{https}: / / \mathrm{n} 9 . \mathrm{cl} / 9 \mathrm{pha}}$ & 94 & 4,0 & $\$ 46.50$ \\
\hline 9. A natomy and Physiology & $\overline{\mathrm{https}: / / \mathrm{n} 9 . \mathrm{cl} / \mathrm{kwq} 2}$ & 67 & 4,3 & $\$ 34.99$ \\
\hline 10. M uscle System Pro iii - iPhone & https://n9.cl/e5z3 & 45 & 3,9 & $\$ 4.99$ \\
\hline 11. T eam Lab Body & https://n9.cl/jg7 & 38 & 4,0 & $\$ 59.00$ \\
\hline 12. $M$ uscle 2 - iPhone Edition & https://n9.cl/aca3 & 26 & 4,0 & 4.49 \\
\hline 13. Palpation Mapp & https://n9.cl/crzt & 23 & 4,7 & $\$ 9.99$ \\
\hline 14. A natomy Quiz Pro & https://n9.cl/tghw & 19 & 3,8 & $\$ 1.99$ \\
\hline 15. M y Incredible Body & https://n9.cl/s31 & 18 & 4,3 & $\$ 24.99$ \\
\hline 16. B rain and Nervous System Pro iii & $\overline{\mathrm{https}: / / \mathrm{n} 9 . \mathrm{cl} / \mathrm{zyl} b}$ & 11 & 3,5 & $\$ 199$ \\
\hline 17. $\mathrm{H}$ eart Pro iii - iPhone & https://n9.cl/1til & 9 & 3,4 & $\$ 4.99$ \\
\hline 18. S keletal Anatomy 3D - Quiz and Reference & https://n9.cl/wgma & 8 & 4,3 & $\$ 2.99$ \\
\hline 19. A tlas de Anatomia Humana & https://n9.cl/n28 & 7 & 5,0 & $\$ 92.90$ \\
\hline 20. A natomyLearning 3D OFFLINE - FULL UNLOCKED & $\mathrm{https}: / / \mathrm{n} 9 . \mathrm{cl} / 227 \mathrm{~h}$ & 7 & 2,1 & $\$ 41.50$ \\
\hline
\end{tabular}




\section{Selección de Apps para el estudio de la Anatomía huma-} na. Los autores de este documento, descargaron las apps clasificadas y las instalaron en dispositivos móviles, se analizaron con detalle sus características, imágenes, idiomas y contenidos temáticos. Se seleccionaron hasta el momento tres apps que podrían ser de gran ayuda a los estudiantes de salud al momento de estudiar anatomía, estas fueron Anatomy learning, Anatomy quiz, y aunque al momento de esta selección, Anatomist se ubicaba en el puesto 24, se tuvo en cuenta para la selección por sus características didácticas. Así mismo, algunos estudiantes compraron y descargaron por su cuenta la aplicación Human Anatomy atlas 2020. Se propuso a todos los estudiantes de este trabajo descargarlas e instalarlas a sus dispositivos y se implementaron durante la parte presencial de las clases, antes y después de ellas.

Las aplicaciones móviles son válidas para el estudio del cuerpo humano. Después de aplicar el instrumento el cual pretendía validar las aplicaciones móviles para estudiar anatomía, se halló lo siguiente:

El $99 \%$ de los estudiantes considera útil el uso de las Apps para el estudio de la Anatomía humana.

Las ventajas que les aportan a los estudiantes las aplicaciones móviles para estudiar anatomía, son las siguientes (Fig. 2):

- El $37 \%$ de las opiniones dice que mejora el aprendizaje porque muchos de estudiantes aprenden anatomía de manera visual.

- El $30 \%$ de las opiniones dice que tienen una fácil visualización porque muestra gráficos en 3D y permite realizar rotaciones en los tres ejes tridimensionales.

- El $21 \%$ de las opiniones dicen que se tiene disponibilidad en cualquier lugar y momento porque al estar la app en un teléfono móvil, este se puede llevar consigo en todo momento lo que permitirá repasar antes de la clase o desde la comodidad de los hogares.

- El $7 \%$ afirma que es una manera diferente de aprender porque se deja atrás los modelos convencionales y se aprovecha en auge de las nuevas tecnologías.

- El $4 \%$ dice que evalúa el conocimiento, porque a través de pequeños quices que realizan algunas apps se puede evidenciar las falencias que se tengan y realizar un proceso de retroalimentación.

Las limitaciones o desventajas que presentan las apps al momento de estudiar anatomía son las que se muestran a continuación (Fig. 2):

- El $30 \%$ de las opiniones dice que tienen limitación con el costo de estas porque una gran parte de las apps tienen paquetes restringidos y otras requieren de un pago para poder realizar su respectiva descarga, además de esto algunas apps solo funcionan si están conectadas a internet lo implicaría un costo adicional a su uso.

- El $23 \%$ de las opiniones dice que las apps generan distracciones en los estudiantes, porque ellos se entretienen fácilmente con las notificaciones de los teléfonos móviles y con los anuncios que estas presentan.

- El $18 \%$ afirma que no se acercan la realidad porque a través de las apps no se puede palpar estructuras, las imágenes aquí presentes son simuladas y no se acercan a lo que es una estructura real.

- El $16 \%$ de las opiniones afirma haber tenido algún tipo de problema técnico con su teléfono móvil al momento de estudiar anatomía porque algunas aplicaciones consumen gran cantidad de espacio de almacenamiento en el teléfono, lo que conlleva al mal funcionamiento del equipo, también han ocurrido que algunas apps no están disponibles en los dos sistemas operativos (IOs y Android).

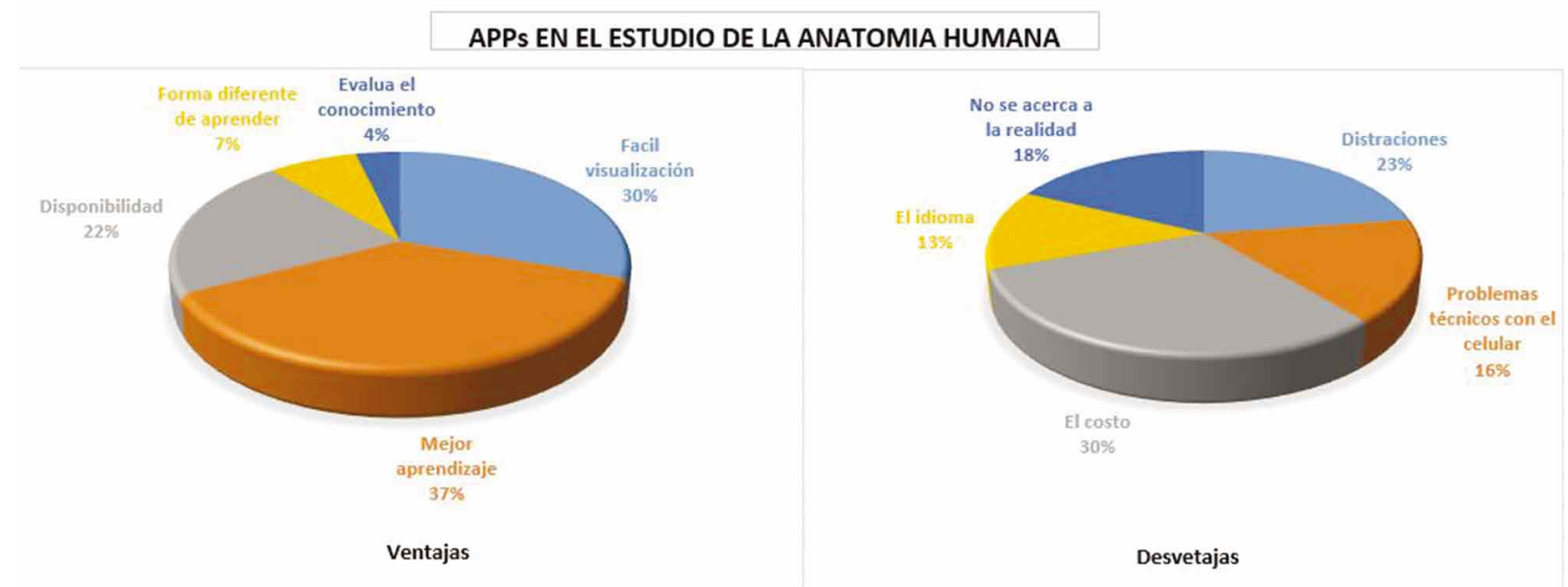

Fig. 2. Ventajas y desventajas de las APPs en el estudio de anatomía humana. 
- El $12 \%$ de las opiniones dice que el idioma es una gran desventaja de las apps, porque algunas de ellas están en inglés y a los estudiantes todavía se les dificulta el uso y comprensión de este lenguaje.

Según la preferencia de los estudiantes en cuanto a seleccionar la mejor de las apps, se tiene la siguiente clasificación:

- El $46 \%$ de los estudiantes prefiere usar Anatomy quiz porque evalúa el conocimiento, es completa e ilustrativa, ayuda a memorizar estructuras y porque tiene una visualización en 3D.

- El $24 \%$ de los encuestados prefiere a Anatomy learning porque es muy completa, brinda una excelente visualización ya que es en 3D, es didáctica y brinda la posibilidad de realizar quices.

- El $16 \%$ de los estudiantes tiene preferencia por la aplicación Anatomist, porque esta tiene variedad de contenido, mejor visualización de estructuras y permite la variación del nivel de aprendizaje.

- El $11 \%$ prefiere Human anatomy atlas 2020, aunque esta aplicación tenga un costo elevado la prefirieron porque está muy completa, permite realizar disección y por su excelente definición.

- El $1 \%$ tiene preferencia por Complete Anatomy.

- El $3 \%$ no tuvo preferencia por ninguna App.

El $99 \%$ de los encuestados recomienda las Apps como un método de complemento para el estudio de la anatomía humana. Recomendarían a otro compañero utilizar Anatomy quiz, Anatomy Learning, Anatomist, Human Anatomy atlas 2020 y Complete Anatomy para el estudio de la Anatomía humana por las siguientes razones:

- El $31 \%$ justifica que es una mejor y diferente estrategia de estudio

- El $24 \%$ informa que tienen quices

- El $19 \%$ dice que brindan una mejor visualización, lo que permite una mejor ubicación de las estructuras

- El $14 \%$ las recomienda por su disponibilidad

- El $12 \%$ argumenta que son una estrategia de repaso de temas vistos en clase $12 \%$

\section{DISCUSIÓN}

En la educación hemos sido testigos de que los recursos didácticos mediados por tecnologías son una alternativa para desarrollar procesos de aprendizaje. Con esto, está claro que el conocimiento no solo se adquiere en un salón de clases (Ramírez Montoya, 2009). Los avances en tecnología han cambiado la forma de enseñanza y aprendizaje de los estudiantes universitarios en las ciencias experimentales y de la salud (Juanes Méndez, 2016).

En el estudio de la Anatomía humana las imágenes son fundamentales para el aprendizaje (Johnson et al.), es importante que además de la teoría los estudiantes puedan observar las estructuras y aún más cuando éstas se presentan de manera dinámica, con movimientos y rotaciones respectivas (Jaffar, 2012; Raikos \& Waidyasekara). Las imágenes que se encuentran en los libros favorecen el aprendizaje. No obstante, una imagen precisa, con recursos multimediales, le permitirá al estudiante acceder a ella en la hora que le parezca más conveniente o en el ritmo de aprendizaje que le favorezca. La utilización de la tecnología es un reto para los profesores que tiene que adaptarse a los nuevos entornos educativos. La tecnología avanza notoriamente y para sacarle provecho la solución no radica en prohibir el uso de esta, sino que, por el contrario, está en buscar alternativas donde de manera favorable se pueda incluir al entorno educativo.

Con este trabajo se evidencia que los dispositivos móviles son importantes para los estudiantes sobre todo para alcanzar su éxito académico(Briz-Ponce \& Juanes-Méndez, 2015). Una App permitirá el repaso de un tema minutos antes de una clase o estudiar para un examen mientras se transporta. Permitirá hacer un proceso de retroalimentación, ya que estas poseen un sistema de evaluaciones que le permite al usuario mirar en que está fallando y aprender de sus equivocaciones.

El aprendizaje autodirigido basado en imágenes dinámicas y apoyado en software de realidad virtual es significativamente mayor en comparación con el aprendizaje de imágenes estáticas convencionales (Castro Alonso, 2019). De allí la importancia deincorporar Apps al estudio de la anatomía. Las ayudas de aprendizaje con procedimientos interactivos, suponen un incentivo para el estudiante (Juanes Méndez). Es por esto que las Apps son un recurso valioso porque brindan un espacio innovador y con mejor visualización, aunque estas tengan algunas limitaciones como el costo o el idioma, estos son pormenores frente a las grandes ventajas que poseen. Finalmente, conocer las aplicaciones móviles en el estudio del cuerpo humano podrá aportar a una mejor enseñanza y comprensión de las temáticas vistas en una clase de Anatomía.

LUCERO, M. J. E. \& ÁLZATE, M. O. A. Mobile applications for the study of human anatomy. Int. J. Morphol., 38(5):1365-1370, 2020.

SUMMARY: The learning through mobile 
applications (Apps) is a subject of great importance that is associated to interact with virtual reality. Despite the large number of Apps available, there is not enough information to guide the usefulness in the didactics of anatomy. That is why this work aims to evaluate the use of mobile applications in the study of human anatomy. Methods were sought for applications for the study of human anatomy, using the App Store and Google Play download platforms. Apps were prioritized giving importance to those that had a greater number of people who had valued it, and the score given by its users. Apps were added to the study of human anatomy in health students at universities in city of Manizales (Colombia). An instrument was applied to evaluate the usefulness in the learning of the different human structures. The search resulted in 113 applications of which 70 are free and 43 have a download cost. Of these, Anatomy quiz is the most preferred by students when studying anatomy. The use of Apps for the study of human anatomy prepares students to face a real structure; unlimited specimens are available for virtual dissection and to develop spatial skills. Knowing the Apps available for the study of the human body contributes to better didactics of anatomy.

KEY WORDS: Mobile applications; Anatomy; Learning; Virtual reality $(\mathrm{MeSH})$.

\section{REFERENCIAS BIBLIOGRÁFICAS}

Alzate-Mejía, O. A. \& Tamayo-Alzate, O. E. Metacognition in the learning of anatomy. Int. J. Morphol., 37(1):7-11, 2019.

Basogain, X.; Olabe, M.; Espinosa, K.; Rouèche, C. \& Olabe, J. C. Realidad aumentada en la educación: una tecnología emergente. 2015. Disponible en: http://files.mediaticos.webnode.es/200000016-a645ea73b3/ realidad\%20A..pdf

Briz-Ponce, L. \& Juanes-Méndez, J. A. Mobile devices and apps, characteristics and current potential on learning. J. Inf. Technol. Res., 8(4):12, 2015. Disponible en: https://www.igi-global.com/article/mobiledevices-and-apps-characteristics-and-current-potential-on-learning/ 145392

Castro Alonso, P. L. Introducción al uso de imágenes digitales en formato web en el aprendizaje de la histología humana. Educ. Med., 20(5):2803, 2019.

De Pedro Carracedo, J. \& Martínez Méndez, C. L. Realidad aumentada. Una alternativa metodológica en la educación primaria Nicaragüense. Rev. Iberoam. Tecnol. Aprendiz., 7(2):102-8, 2012.

Farfán, C. E.; Schneeberger, L. D.; Besa, H. J.; Salgado, A. G. \& Inzunza, H. O. Web service anatomy: report of an educational experience. Int. J. Morphol., 34(1):136-42, 2016.

Jaffar, A. A. YouTube: an emerging tool in anatomy education. Anat. Sci. Educ., 5(3):158-64, 2012.

Johnson, E. O.; Charchanti, A. V. \& Troupis, T. G. Modernization of an anatomy class: from conceptualization to implementation. A case for integrated multimodal-multidisciplinary teaching. Anat. Sci. Educ., 5(6):354-66, 2012.

Juanes Méndez, J. A. Estado actual de las nuevas tecnologías en la enseñanza de las ciencias experimentales y de la medicina en particular. Educ. Med., 17(1):1-2, 2016.
López García, M. \& Morcillo Ortega, J. G. Las TIC en la enseñanza de la Biología en la educación secundaria: los laboratorios virtuales. Rev. Electron. Enseñ. Cienc., 6(3):562-76, 2007.

Raikos, A. \& Waidyasekara, P. How useful is YouTube in learning heart anatomy? Anat. Sci. Educ., 7(1):12-8, 2014.

Ramírez Montoya, M. S. Recursos tecnológicos para el aprendizaje móvil (mlearning) y su relación con los ambientes de educación a distancia: implementaciones e investigaciones. RIED, 12(2):57-82, 2009.

Dirección para correspondencia:

Oscar Andrés Álzate Mejía

Departamento de Ciencias Básicas Biológicas

Universidad Autónoma de Manizales

Manizales

COLOMBIA

Email: oalzate@autonoma.edu.co

Recibido : 11-03-2020

Aceptado: 03-05-2020 